\title{
Functional assessment of foot and ankle tendinopathies treated with tendoscopy
}

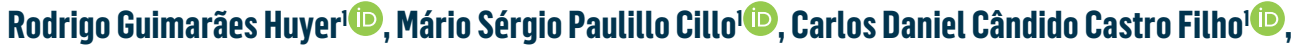 \\ Hallan Douglas Bertelli' ${ }^{10}$, Renato Morelli Berg' ${ }^{10}$ \\ 1. Orthopedics and Traumatology Departament PUC-Campinas, Campinas, SP, Brazil.
}

\begin{abstract}
Objective: To assess postoperative clinical functional outcomes, based on the American Orthopaedic Foot \& Ankle Society (AOFAS) score, of tendoscopies performed in the treatment of foot and ankle pathologies.

Methods: Our comparative assessment used AOFAS scores obtained preoperatively and at early and late postoperative stages - 1 month and 6 to 12 months after surgery - of 14 patients with foot and ankle tendinopathies. These included peroneal tendon dislocation, peroneal tendonitis, and tearing of the peroneus longus or brevis, all treated with tendoscopy for peroneal reconstruction and tenorrhaphy. The AOFAS score was obtained by functional assessment during outpatient physical examination. We presented a descriptive analysis of cases, comparing scores over time through the Friedman test followed by Dunn's test. The relationship between score variations and sex was assessed using the Mann-Whitney test; their comparison with age used Spearman's linear correlation coefficient. Significance levels were $5 \%$.
\end{abstract}

Results: The AOFAS score showed important improvements such as preoperative scores of 56 and 67 followed by postoperative scores of 100 both in the early and late stages, supporting the efficacy and persistence of this treatment strategy. The p-value obtained after statistical analysis was $<0.0001$.

Conclusion: We concluded that the treatment of foot and ankle comorbidities with tendoscopy, in addition to being less invasive, shows consistency and efficacy as demonstrated by the AOFAS score and functional assessment via postoperative physical examinations. AOFAS scores were increased and maintained at high levels in the postoperative period, demonstrating the efficacy of this procedure and the duration of treatment results.

Level of Evidence IV; Case Series; Therapeutic Studies - Investigation of Treatment Results.

Keywords: Tendinopathy; Ankle injuries; Foot injuries; Tendon injuries; Surgical procedures, operative.

\section{Introduction}

In the orthopaedic environment, the evolution of treatment of foot and ankle comorbidities has become increasingly evident. Professionals in this specialty frequently observe important improvements in prognosis when the proposed treatment consists in a less invasive and more efficient alternative ${ }^{(1-5)}$. In this study, tendoscopy was used as a treatment strategy for foot and ankle comorbidities such as peroneal tendon dislocation, tearing, or tendonitis to prove its efficacy. The idea of the study consists in strengthening, within the orthopaedic scenario of the foot and ankle specialty, less invasive approaches that provide good and long-lasting results. The objective of this research was to report, by assessing 14 patients, improvements in symptoms after tendoscopic treatment by using the American Orthopaedic Foot \& Ankle Society (AOFAS) score - evaluating pain, functionality, stability, mobility, and alignment of the hindfoot - in the preoperative (PRE), early postoperative (ePO, 1 month), and late postoperative (IPO, 6-12 months) periods. The intention is to prove the efficacy of less invasive treatments aiming at substituting - when possible - more invasive treatments that
Study performed at the Orthopedics and Traumatology Departament PUCCampinas, Campinas, SP, Brazil.

Correspondence: Renato Morelli Berg. Rua Presidente João Goulart, 532, Jardim Chapadão - 13070-060, Campinas, SP, Brazil. E-mail: rmorelliberg@gmail.com. Conflicts of Interest: none. Source of funding: none. Date received: April 14, 2021. Date accepted: July 11, 2021. Online: August 31, 2021.
How to cite this article: Huyer RG, Cillo MSP, Castro Filho CDC, Bertelli HD, Berg RM. Functional assessment of foot and ankle tendinopathies treated with tendoscopy. J Foot Ankle. 2021;15(2):128-32. 
show unfavorable progressions regarding wound healing, higher chances of infection, and longer recovery periods after surgery, which could provide the specialty with better prognoses and be of utmost importance for the evolution of treatment strategies and patient wellbeing.

\section{Methods}

This study was approved by the ethics committee of Hospital Pontifícia Universidade Católica (PUC) - Campinas and was performed via a case series analysis with a retrospective clinical design. The study aimed to assess 14 patients. Inclusion criteria considered male and female patients of all ages who were diagnosed with tendon pathologies surrounding the foot and ankle, with the possibility of being treated by tendoscopy. Exclusion criteria consisted of patients who denied participation in the study, who presented signs of active infection in a previous surgical site, who had lower limb deformities that could limit rehabilitation, after-effects of comorbidities unrelated to the tendon injuries observed in this study, or neurological alterations. After triage and inclusion of the participant, assessment was performed by applying the AOFAS score(6) in the outpatient clinic of the Department of Orthopaedics and Traumatology of Hospital PUC - Campinas, at preoperative and postoperative (early, 1 month; late, 6-12 months) periods. The assessment, made through the application of questionnaires and a clinical functional examination, took around 30 minutes and were divided into 3 stages: anamnesis, questionnaire application, and recording of the obtained result. The free and informed consent form presented, as the objective explained to the patients, the functional assessment of postoperative results based on the AOFAS scores of tendoscopies performed in the treatment of foot and ankle pathologies.

Our methodology consisted in the descriptive analysis, through position and dispersion measures such as median, minimum, and maximum values for continuous variables, and frequency tables (absolute and relative) for categorical variables. For comparing scores over time, we applied the Friedman test followed by Dunn's test for identifying differences. For assessing the relationship between score variations and sex, we used the Mann-Whitney test. For comparing them with age, we used Spearman's linear correlation coefficient. The significance level adopted for the statistical tests was $5 \%$.

Statistical tests applied in this study were non-parametric due to the reduced sample size. The level of significance adopted for the tests was $5 \%$ and analyses were performed using the SAS System software for Windows ${ }^{(7)}$.

\section{Results}

According to our statistical analyses, by correlating comorbidities and treatments (Table 1), we noted an important evolution in AOFAS scores obtained in the PRE, ePO, and IPO periods. AOFAS scores obtained in the ePO and IPO periods were significantly different from those in the PRE period, demonstrating an interesting efficacy of the tendoscopy treat-

Table 1. General descriptive analysis

\begin{tabular}{|c|c|c|c|c|c|c|}
\hline \multicolumn{4}{|l|}{ Sex } & Frequency & Percentage & Cumulative frequency \\
\hline \multicolumn{4}{|l|}{$f$} & 7 & 46.67 & 7 \\
\hline \multicolumn{4}{|l|}{$\mathrm{m}$} & 8 & 53.33 & 15 \\
\hline \multicolumn{4}{|l|}{ Comorbididy } & Frequency & Percentage & Cumulative frequency \\
\hline \multicolumn{4}{|l|}{ Gout } & 1 & 6.67 & 1 \\
\hline \multicolumn{4}{|c|}{ Peroneal dislocation } & 6 & 40.00 & 7 \\
\hline \multicolumn{4}{|c|}{ Pes cavus + peroneus brevis tear } & 1 & 6.67 & 8 \\
\hline \multicolumn{4}{|c|}{ Peroneus longus tear } & 1 & 6.67 & 9 \\
\hline \multicolumn{4}{|c|}{ Peroneal tendonitis } & 5 & 33.33 & 14 \\
\hline \multicolumn{4}{|c|}{ Peroneal tendonitis + retromalleolar peroneus brevis split tear } & 1 & 6.67 & 15 \\
\hline \multicolumn{4}{|l|}{ Procedure } & Frequency & Percentage & Cumulative frequency \\
\hline \multicolumn{4}{|c|}{ Tendoscopy + Peroneal reconstruction } & 6 & 40.00 & 6 \\
\hline Tendoscopy + & ion & & & 1 & 6.67 & 7 \\
\hline Tendoscopy + & sis - & calcan & & 1 & 6.67 & 8 \\
\hline Posterior tibia & and & & & 1 & 6.67 & 9 \\
\hline Peroneal tend & & & & 5 & 33.33 & 14 \\
\hline Tendoscopy + & & & & 1 & 6.67 & 15 \\
\hline Variable & $n$ & Mean & SD & & & Maximum \\
\hline AOFAS_PRE & 15 & 60.6 & 6.7 & & & 69.0 \\
\hline AOFAS_PO1 & 15 & 93.7 & 4.8 & & & 100.0 \\
\hline AOFAS_6_12 & 13 & 96.7 & 5.7 & & & 100.0 \\
\hline
\end{tabular}


ment proposed by this study. Moreover, no significant difference was observed between postoperative scores ( $p$-value $<0.0001$ between ePO and IPO - Friedman test) (Table 2), which highlights the duration of treatment effectivity.

The AOFAS scores obtained during the preoperative and postoperative periods showed important increases, indicating important improvements in prognosis considering the patient's pain and functionality. This was done using a physical examination for assessing stability of the operated limb, its function, mobility, and hindfoot alignment. Preoperative scores varied from 47 to 69 , while postoperative scores varied from 89 to 100 in the ePO period and from 85 to 100 in the IPO period (Figure 1). These results were positively significant.

Another interesting and positive aspect was in regard to the age and sex of patients, since no significant differences were observed between different groups; this emphasized the universality of treatment by tendoscopy.

This way, we could contribute positively to the purpose of this study, which was to foster the idea of good prognoses and the efficacy of the tendoscopic treatment of foot and ankle comorbidities, aiming to render it increasingly accepted and used in the orthopaedic environment.

\section{Discussion}

Arthroscopy of the foot and ankle is a common technique. With the emergence of new, more refined surgical techniques and arthroscopic instrumentation, there was a natural transition to tendoscopic techniques, and this surgical modality has been incorporated to the arsenal of the foot and ankle surgeon. Tendoscopy is an endoscopy of the tendon sheath that has been described in many tendons of the foot and ankle, including the posterior tibial, peroneal tendons, the flexor digitorum longus, anterior tibial, and Achilles tendons ${ }^{(1-5)}$. In this study, we mainly analyzed the treatment of peroneal tendon comorbidities, due to a higher incidence among the selected patients.

The conservative treatment of extra-articular ankle pathologies is successful in most cases, but non-surgical treatment has a failure rate of $10 \%$ to $25 \%^{(2)}$. When clinical treatment fails, conventional open surgery can be indicated. As opposed to the elbow or the knee, for example, posterior tendon structures of the ankle are deep and can be difficult to feel at palpation; in addition, these structures are very close together, which could hamper diagnostic imaging ${ }^{(8)}$. When trea- ting posterior extra-articular ankle pathologies, posterolateral or posteromedial incisions are made, which could imply in a risk of damage to adjacent structures ${ }^{(2-5,8)}$ Complication rates of open surgery procedures of the Achilles tendon vary between $4.7 \%$ and $11.6 \%$; wound infection, dehiscence, and pain are the most frequently described problems ${ }^{(2)}$. With larger incisions, postoperative care may include immobilization in order to promote better wound healing ${ }^{(8)}$. The aim of this study relies in the proposition of shorter rehabilitation periods to patients, thus proving the efficacy of the tendoscopic treatment and its long-lasting effect, questioning the need for choosing invasive treatments that require larger incisions and have higher chances of complications and longer recovery periods.

Tendoscopies performed around the ankle joint, especially posterior procedures, may be technically demanding but offer a unique perspective of the pathological processes involving these structures. Certain anatomical particularities may render tendoscopy challenging. Factors that hinder the identification of structures or the access to them, such as decreases in tendon volume due to severe chronic injury or stenosing tenosynovitis or restricted access due to the curvature of the tendon's course, could prevent access to the site of the pathology. Therefore, when performing a tendoscopy, the sur-

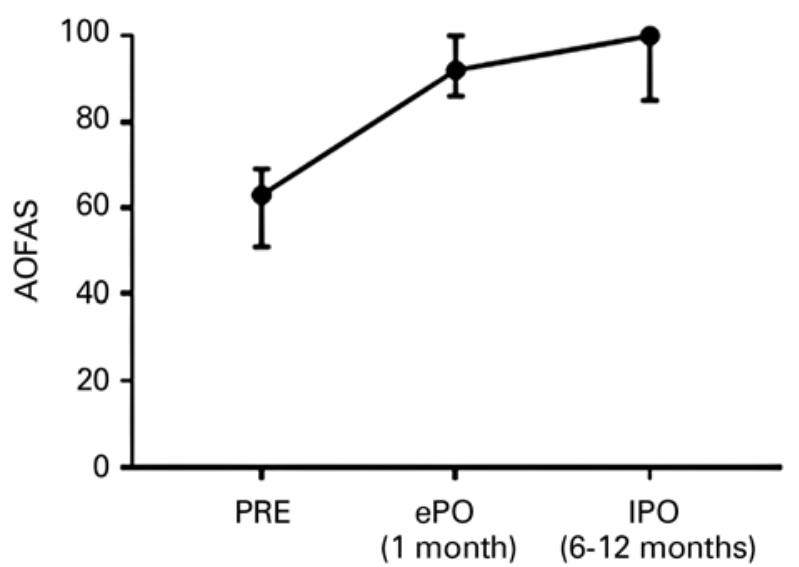

Figure 1. Median American Orthopaedic Foot \& Ankle Society (AOFAS) scores for each period. Vertical bars indicate variations and minimum and maximum values.

Table 2. Descriptive analysis and comparison of the American Orthopaedic Foot \& Ankle Society (AOFAS) scores between periods

\begin{tabular}{llllccc}
\hline Variable & $\mathbf{n}$ & Mean & SD & Minimum & Median & Maximum \\
\hline AOFAS_PRE & 13 & 62.0 & 5.7 & 51.0 & 63.0 & 69.0 \\
\hline AOFAS_PO1 & 13 & 93.5 & 5.1 & 86.0 & 92.0 & 100.0 \\
\hline AOFAS_6_12 & 13 & 96.7 & 5.7 & 85.0 & 100.0 & 100.0 \\
\hline
\end{tabular}

p-value (Friedman test) $<\mathbf{0 . 0 0 0 1}$, differences between (Dunn's test): preoperative and postoperative ( 1 month) periods; preoperative and postoperative (6-12 months) periods. No difference between 1 month and 6-12 months after surgery. 
geon should be familiarized with the anatomy of the foot and ankle.

Some studies aimed to widen the possibility of knowing the tendoscopic view by the foot and ankle orthopedist. Van Dijk and Kort $^{(5)}$ performed a study with cadavers where, in 3 out of 7 tendoscopic investigations, they found that the peroneal tubercle on the calcaneus was between peroneal tendons, 4 to $5 \mathrm{~cm}$ distal from the tip of the fibula. The posterior tibial tendon has myotendinous connections throughout its course. Van Dijk and Kort ${ }^{(5)}$ have published a review on the anatomy of the posterior tibial tendon, and Steenstra and Van Dijk ${ }^{(9)}$ have shown a general view of the Achilles tendon. Lui et al. ${ }^{(10)}$ studied the flexor hallucis tendon and its various segments (zones). In this study, the authors verified that the medial plantar nerve was on the medial side of the plantar region, proximal to the tendon sheath in all samples; its relationship with the more distal sheath was variable. Synovectomy at this site should be performed with caution due to the anatomic proximity of the tarsal tunnel structures. The tendoscopy of posterior tibial, peroneus longus and brevis, and flexor hallucis longus tendons was described by van Dijk et al. ${ }^{(5,8)}$. Maquirriain ${ }^{(11)}$ presented a study with 9 cadavers in which he showed that adequate access to the Achilles tendon could be achieved via an endoscope. Samarco and Henning ${ }^{(12)}$ described the arthroscopic treatment of the peroneus tertius.

Sammarco(13) began recommending, as routine, the tendoscopy of peroneal tendons during the reconstruction of the ankle ligament complex in case of suspicion of peroneal pathologies.

Lui ${ }^{(14)}$ stated that, with solid knowledge of indications, merits, and potential risks of new techniques, endoscopic procedures will be powerful tools in foot and ankle surgery and this study intended to provide further information and contribute so that tendoscopy is increasingly present in the daily practice of the foot and ankle orthopedist, effectively showing its good prognostic and the duration of its results. Furthermore, we have observed the universality of this treatment, which can be recommended for men or women of different ages.
Monteagudo et al. ${ }^{(15)}$ state that tendoscopy is an apparently safe and reliable procedure for treating some foot and ankle disorders, and it can be used as an adjuvant procedure to other techniques. Level IV and $V$ studies are predominantly found in the literature, and level I studies are not yet available. However, owing to the existence of many promising studies and constantly evolving endoscopic techniques for treating tendinopathies of the foot and ankle, the procedure may be recommended and more studies with higher levels of evidence should be promoted in order to strengthen this procedure in the treatment of foot and ankle injuries. This study has a level of evidence IV, which is a case series study that aims to broaden the idea of reliability and safety of the tendoscopic treatment even further, in addition to its efficacy.

\section{Conclusion}

The endoscopic treatment of the foot and ankle, by principle, aims to provide, in a less invasive manner, relief to symptoms of some foot and ankle comorbidities, such as peroneal tendon dislocation, peroneal tendonitis, or tearing of the peroneus longus or brevis, which are some of the pathologies treated in this study.

The objective of showing and underlining the efficacy of tendoscopy and the maintenance of its good prognosis by using the AOFAS score during the clinical functional assessment performed in postoperative periods was successfully achieved. AOFAS scores were significantly positive, remaining above 86 in the recent and late postoperative periods and reaching 8 values of 100 (best possible result for the score) in the late postoperative period. Moreover, the idea or choosing a less invasive procedure, even if requiring superior technique and instrumentation, was reinforced with great results through a reduction in the postoperative recovery period, the maintenance of symptom relief, and functionality recovery by patients subjected to functional assessment, whether they were women or men, of various ages, which confers an interesting universality to this treatment.

Authors' contributions: Each author contributed individually and significantly to the development of this article: RGH *(https://orcid.org/0000-0003-39518408) Conceived and planned the activities that led to the study, bibliographic review, participated in the review process and approved the final version; MSPC *(https://orcid.org/0000-0002-0758-2547) Conceived and planned the activities that led to the study, bibliographic review, participated in the review process and approved the final version; CDCCF *(https://orcid.org/0000-0003-3522-1076) Performed the surgeries, data collection and approved the final version; HDB *(https://orcid.org/0000-0002-1901-3309) Performed the surgeries, data collection and approved the final version; RMB *(https:// orcid.org/0000-0002-4218-3023) Interpreted the results of the study, participated in the review process, data collection and formatting of the article. All authors read and approved the final manuscript. * ORCID (Open Researcher and Contributor ID) (iD). 


\section{References}

1. Bulstra GH, Olsthoorn PG, Niek van Dijk C. Tendoscopy of the posterior tibial tendon. Foot Ankle Clin. 2006;11(2):421-7.

2. Morag G, Maman E, Arbel R. Endoscopic treatment of hindfoot pathology. Arthroscopy. 2003;19(2):E13

3. van Dijk CN. Hindfoot endoscopy for posterior ankle pain. Instr Course Lect. 2006;55:545-54.

4. van Dijk CN. Hindfoot endoscopy. Foot Ankle Clin. 2006;11(2):391-414.

5. van Dijk CN, Kort N. Tendoscopy of the peroneal tendons. Arthroscopy. 1998;14(5):471-8.

6. Rodrigues RC, Masiero D, Mizusaki JM, Imoto AM, Peccin MS, Cohen $\mathrm{M}$, et al. Translation, cultural adaptation and validity of the "American Orthopaedic Foot and Ankle Society (AOFAS) Ankle-Hindfoot Scale". Acta Ortop Bras. 2008;16(2):107-11.

7. SAS System for Windows (Statistical Analysis System), versão 9.4. SAS Institute Inc, 2002-2012, Cary, NC, USA.

8. van Dijk CN, Scholten PE, Krips R. A 2-portal endoscopic approach for diagnosis and treatment of posterior ankle pathology. Arthroscopy. 2000;16(8):871-6.
9. Steenstra F, van Dijk CN. Achilles tendoscopy. Foot Ankle Clin. 2006;11(2):429-38.

10. Lui TH, Chan KB, Chan LK. Cadaveric study of zone 2 flexor hallucis longus tendon sheath. Arthroscopy. 2010;26(6):808-12.

11. Maquirriain J. Endoscopic release of Achilles peritenon. Arthroscopy 1998;14(2):182-5.

12. Sammarco GJ, Henning C. Peroneus tertius muscle as a cause of snapping and ankle pain: a case report. Am J Sports Med. 2007;35(8):1377-9.

13. Sammarco VJ. Peroneal tendoscopy: indications and techniques Sports Med Arthrosc Rev. 2009;17(2):94-9.

14. Lui TH. Arthroscopy and endoscopy of the foot and ankle: indications for new techniques. Arthroscopy. 2007;23(8): 889-902.

15. Monteagudo M, Maceira E, Martinez de Albornoz P. Foot and ankle tendoscopies: current concepts review. EFORT Open Rev. 2017;1(12):440-7. 